\title{
PREVENDO A INSOLVÊNCIA DE OPERADORAS DE PLANOS DE SAÚDE
}

PREDICTING HEALTH PLAN INSOLVENCY

\section{RESUMO}

Dada a relevância das operadoras de planos de saúde na prestação de serviços de saúde e sua grande importância para clientes individuais e empresariais, assim como para profissionais e empresas prestadoras de serviços de saúde, é importante antecipar a capacidade financeira das operadoras que efetivamente cumprir suas obrigações contratuais (prover serviços a clientes, pagar prestadores). Para isso, desenvolvemos um modelo de previsão de insolvência específico para operadoras de planos de saúde. Por meio de uma regressão logística sobre 17 indicadores financeiros de cerca de 600 operadoras brasileiras de planos de saúde, desenvolvemos um modelo capaz de prever a insolvência de uma operadora após um ano, e analisamos a precisão desse modelo específico em comparação com a de um modelo geral bastante popular, o escore Z" de Altman.

\section{André Luiz de Souza Guimarães}

Administrador do Banco Nacional de Desenvolvimento Econômico e Social - RJ, Brasil

alsguimaraes@gmail.com

\section{Washington Oliveira Alves}

Especialista em Regulação da Agência Nacional de Saúde Suplementar - RJ, Brasil

washington.alves@gmail.com

Recebido em 31.03.2008. Aprovado em 08.07.2009

Avaliado pelo sistema double blind review

Editores Científicos: Organizadores do Fórum

ABSTRACT Given the relevance of health plans in the provision of healthcare and their high importance for individual and business customers, as well as healthcare professionals and companies, it is important to predict financial capacity of health plans to effectively fulfill their obligations. We develop an insolvency prediction model specific for health plans. Through a logistic regression of 17 financial measures of around 600 Brazilian health plan carriers, we develop a one-year insolvency prediction model, and its accuracy is analyzed and contrasted to that of a generic corporate model, Altman's Z" score.

PALAVRAS-CHAVE Insolvência, gestão da saúde, planos de saúde, capacidade financeira em saúde, regressão logística. KEYWORDS Insolvency, healthcare management, health plans, healthcare financial capacity, logistic regression. 


\section{INTRODUÇÃO}

Em entrevista à Gazeta Mercantil de 21 de janeiro de 2005, Fausto Pereira dos Santos, presidente da Agência Nacional de Saúde Suplementar (ANS), órgão responsável pela regulação do mercado brasileiro de planos de saúde, afirmou que cerca de 300 operadoras de planos de saúde (OPS) enfrentam dificuldades financeiras significativas. Esse cenário, de certa forma, passou a ficar mais evidente após a efetiva regulação do setor, depois da Lei $\mathrm{n}^{\circ}$ 9.656/98; entretanto, não é tão diferente da situação vivenciada por outros sistemas privados de saúde no mundo. De 1980 a 1990, 180 OPS quebraram (CHRISTIANSON, WHOLEY e outros, 1991) nos Estados Unidos. No fim dos anos 1990, por exemplo, hospitais e médicos de Nova Jersey sofreram perdas de cerca de US\$ 150 milhões de dólares com a quebra de duas OPS.

Considerando o forte aumento nos custos da saúde e o aumento da utilização dos serviços devido ao envelhecimento da população (que não são necessariamente os principais motivos do crescimento da insolvência de OPS, mas dificultam sua reversão), essa tendência de crescimento da insolvência de OPS está longe de mudar, e é prudente estar atento à saúde financeira das OPS, principalmente ao considerar os impactos econômicos e sociais da insolvência nas famílias atendidas por empresas insolventes ou à beira da insolvência.

Embora existam alguns estudos de previsão de insolvência de empresas de planos de saúde, os modelos anteriores baseiam-se fundamentalmente nas características do plano, do mercado e da regulação, não incluindo uma ampla variedade de indicadores financeiros. Este estudo toma um caminho diferente, partindo de um conjunto completo de indicadores financeiros de operadoras de plano de saúde brasileiras, para os anos de 2004 a 2008; identificando aqueles indicadores mais significativos na análise da solvência de uma operadora, e desenvolvendo um modelo de regressão logística capaz de estimar a probabilidade de insolvência em um ano (PI) de uma operadora de plano de saúde. O desempenho do modelo desenvolvido é comparado ao desempenho do conhecido modelo de previsão de insolvência aplicado a empresas abertas de qualquer setor - o escore Z" de Altman - a fim de se avaliar a importância de um modelo específico para OPS em face de um modelo genérico.

A análise é realizada com base em uma amostra de 706 OPS brasileiras referentes a 2004, inclusive 28 insolventes (que se tornaram insolventes em 2005). Seguindo essa breve introdução, a próxima seção apresenta uma revisão da literatura relevante sobre metodologias de previsão de ruína. Primeiro, apresentamos e justificamos a escolha de uma regressão logística para desenvolver um modelo de avaliação de risco de insolvência específico para a saúde suplementar. Em seguida, apresentamos uma visão geral e uma análise dos estudos mais recentes sobre insolvência em operadoras de planos de saúde. Na seção seguinte, elaboramos a metodologia utilizada, descrevendo a amostra, os ajustes efetuados, as variáveis utilizadas e o método de análise empregado. A quinta seção apresenta o modelo de regressão logística para prever a insolvência de uma OPS em um ano. Na sexta seção, esse modelo é discutido, validado e comparado ao escore Z" de Altman - um modelo geral bem conhecido e amplamente utilizado. A seção 7 apresenta as conclusões do estudo, ressaltando o valor do desenvolvimento de um modelo específico, para estimar a probabilidade de insolvência, em um ano, de uma OPS.

\section{REVISÃO DA LITERATURA}

Esta seção revê a literatura relevante sobre a previsão de insolvência de operadoras de planos de saúde. Após a primeira análise dos métodos estatísticos mais utilizados para desenvolver modelos de previsão de insolvência, discutimos obras que têm investigado o problema da modelagem da probabilidade de dificuldades financeiras para empresas que comercializem planos de saúde. Importante notar que em toda a literatura sobre previsão de insolvência os autores têm utilizado vários termos para se referir ao mesmo problema fundamental: a incapacidade de honrar as obrigações. Para efeito do presente estudo, os termos: insolvência, quebra, ruína, falência e liquidação são equivalentes. Finalmente, fazemos uma breve introdução à metodologia CAP - cumulative accuracy profile - utilizada nesse estudo para a comparação do desempenho de dois modelos de previsão de insolvência.

\section{ESTUDOS DE PREVISÃO DE INSOLVÊNCIA}

Os estudos de previsão de insolvência têm uma longa tradição, que remonta ao final dos anos 1930 (FITZPATRICK, 1932), e trabalhos empíricos na década de 1960, com as seminais obras de Beaver (1966) e Altman (1968), que desenvolveram modelos univariados e multivariados de previsão de insolvência, utilizando um conjunto de indicadores financeiros. Beaver (1966) utilizou um teste de classificação dicotômica para determinar as margens de erro que o investidor apuraria quando classificasse as empresas, com base em indicadores financeiros individuais, 
como insolventes ou solventes. Ele utilizou uma amostra pareada que combinava 14 indicadores financeiros de 158 empresas (79 insolventes e 79 solventes).

A fim de resolver os problemas verificados na análise univariada de Beaver (1966), Altman (1968) utilizou a análise discriminante múltipla (AMD, em inglês: multiple discriminant analysis - MDA) e avaliou um perfil financeiro mais completo de 66 fábricas (33 solventes e 33 insolventes) que apresentaram pedido de falência entre os anos de 1946 e 1965. Altman examinou 22 indicadores financeiros potencialmente relevantes em cinco categorias - liquidez, rentabilidade, alavancagem, solvência e atividade - e desenvolveu um modelo composto por cinco variáveis que, em conjunto, apresentavam o melhor poder de previsão de insolvência das empresas analisadas.

Após o estudo de Altman, a análise múltipla de discriminantes tornou-se o método estatístico mais utilizado no desenvolvimento de modelos de previsão de insolvência. No entanto, na maioria dos estudos posteriores ao trabalho inicial de Altman, os autores apontam alguns problemas com a AMD (ALTMAN e SABATO, 2007). Primeiro, dois pressupostos básicos da AMD são frequentemente violados quando aplicados a problemas de previsão de insolvência: como a normalidade multivariada e a igualdade de variâncias e covariâncias. Além disso, em alguns modelos da AMD, os coeficientes normalizados não podem ser interpretados como as "inclinações" de uma equação de regressão e, portanto, não indicam a importância relativa das diferentes variáveis.

Considerando as dificuldades associadas à AMD, Ohlson (1980) desenvolveu o primeiro modelo de regressão logística condicional para previsão de insolvência. Os benefícios práticos da regressão logística são hipóteses menos restritivas que as da AMD e um método que permite trabalhar com amostras desproporcionais ou não pareadas (não requer que o número de empresas insolventes na amostra seja igual ao número de empresas solventes). Ohlson utilizou uma série de dados coletados do banco de dados Compustat, que incluía 105 empresas insolventes e 2.058 empresas solventes, com dados de 1970 a 1976. Sua análise levou em consideração 7 indicadores financeiros frequentemente citados na literatura (Log [ativo total], exigível total/ativo total, capital de giro/ativo total, liquidez corrente, lucro líquido/ativo total, receita operacional/ exigível total, e um outro indicador de nível de lucratividade), e 2 variáveis binárias (se houve resultado líquido negativo durante os dois últimos anos; e se o patrimônio líquido é negativo). No entanto, o grau de acerto da classificação do modelo de Ohlson foi inferior ao relatado em estudos anteriores baseados na AMD (ALTMAN, 1968; ALTMAN, HALDEMAN e outros, 1977).
Apesar do baixo desempenho do modelo, Ohlson apresentou fortes argumentos em favor da regressão logística em face da AMD. Do ponto de vista estatístico, a regressão logística se adapta bem às características dos modelos de previsão de insolvência, nos quais a variável dependente é dicotômica (solvente/insolvente) e onde os grupos são discretos, não se sobrepõem e são identificáveis. Além disso, um modelo logit retorna uma pontuação que varia entre zero e um, e reflete de forma conveniente a probabilidade de insolvência da empresa. Por fim, os coeficientes estimados podem ser interpretados como a importância ou contribuição de cada uma das variáveis independentes para a explicação da variável dependente (a probabilidade estimada de insolvência). Após o trabalho de Ohlson (1980), muitos trabalhos acadêmicos passaram a utilizar modelos de regressão logística para prever insolvências (ALTMAN e SABATO, 2007).

Vale ressaltar que o modelo de escore $Z$ de Altman (ALTMAN, 1968; ALTMAN, HALDEMAN e outros, 1977; ALTMAN, BAIDYA e outros, 1979; ALTMAN e HOTCHKISS, 2006) ainda é amplamente utilizado, sendo possivelmente um dos modelos mais difundidos. Neste estudo, adotamos a variante do modelo de Altman conhecida como Z", que estima a probabilidade de uma empresa privada não manufatureira tornar-se insolvente no período de um a dois anos. O modelo também foi aplicado com sucesso a mercados emergentes (ALTMAN, HARTZELL e outros, 1995, 1997), e é calculado a partir da fórmula: Z" $=6,56 X_{1}+3,26 X_{2}+6,72 X_{3}+1,05 X_{4}$, onde: $X_{1}=$ capital de giro/ativo total; $\mathrm{X}_{2}=$ lucros acumulados/ativo total; $\mathrm{X}_{3}=$ Lucro antes de impostos e taxas/ativo total; e $\mathrm{X}_{4}$ = patrimônio líquido/ativo total. Nesse modelo, valores abaixo de 1,1 indicam alto risco de insolvência, e valores acima de 2,6 indicam um baixo risco de insolvência, enquanto valores entre 1,1 e 2,6 representam uma área cinzenta (incerteza).

\section{Estudos de insolvência de empresas de planos de saúde}

A literatura especializada nacional carece de modelos de previsão de insolvência com foco em operadoras de planos de saúde. A literatura internacional, por sua vez, concentra-se nas health maintenance organizations (HMOs) dos Estados Unidos, que - como observado pelos revisores anônimos - apresentam peculiaridades no seu funcionamento em relação às OPS brasileiras, embora ambas as modalidades provejam acesso a tratamento de saúde. Entretanto, mesmo consideradas as diferenças estruturais entre OPS e HMOs e as políticas públicas de saúde dos dois países, a revisão da literatura internacional ainda é 
informativa, pois: 1) mostra que estudos desta natureza têm uma tradição já bem conhecida, e 2) auxilia na identificação de variáveis que possam confirmar sua capacidade preditiva no contexto brasileiro.

Hillman, Pauly e Kerstein (1989), estudando empresas de planos de saúde em operação em 1986, estimaram o ponto de equilíbrio, ou break-even, de uma OPS por meio de regressão logística. Descobriram que duas variáveis de incentivo financeiro estavam positivamente relacionadas ao ponto de equilíbrio das empresas: a exposição dos médicos ao custo dos exames de pacientes em consulta e a dependência do médico em relação à OPS (medida como percentagem de pacientes dos médicos cobertos por uma OPS). A idade e o número de beneficiários também estavam positivamente relacionados ao ponto de equilíbrio, enquanto a forma organizacional de associações de prática independente (na qual os prestadores são contratados de forma independente, sem vínculo trabalhista) estava negativamente relacionada ao ponto de equilíbrio.

Stone e Heffernan (1989) relataram e analisaram os resultados de uma pesquisa realizada em 1988 por um grupo de trabalho da National Association of Insurance Comissioners (NAIC) - Associação Nacional das Agências Reguladoras de Seguros dos Estados Unidos - dedicado ao estudo de insolvência, em que os reguladores foram convidados a apresentar os principais motivos de insolvência de OPS. As razões mais frequentemente citadas por ordem decrescente de importância foram: falta de capital inicial e patrimônio, falta de controle de utilização dos serviços, preços inadequados, insuficiência de capital permanente e lucro, além de uma estimativa inadequada de sinistros ocorridos e não avisados. Stone e Heffernan traçaram o perfil de uma operadora insolvente como uma associação de prática independente com fins lucrativos, em operação há menos de três anos e com menos de 17 mil beneficiários.

Wholey, Christianson e Sanchez (1990) examinaram a relação entre regulação estadual e o desenvolvimento de OPS em cada estado americano, no período de 1979 a 1987. Verificaram a relação entre a probabilidade de saída de uma OPS das áreas metropolitanas e o número real de saídas em função da regulação estatal, das características da comunidade e do efeito da concorrência. Os resultados demonstraram que regulamentações que exigem que os empregadores contratem planos de saúde, as regras de exigências de qualidade, tamanho da comunidade e dos empregadores, e o aumento da concorrência a partir de um número maior de OPS operando na mesma região metropolitana relacionam-se positivamente com a saída de empresas. Por outro lado, a concorrência entre médicos, o percentual da população coberta por OPS e as poucas restrições para contratação demonstraram-se negativamente relacionados com a saída das empresas.

Ahern (1991), analisando a experiência de insolvência de OPS na Flórida, afirmou que muitas falências são causadas por gestões amadoras e intensa competição de preços por uma maior participação de mercado. Ela também tentou avaliar a capacidade preditiva dos indicadores financeiros estimando uma regressão entre os índices financeiros e a rentabilidade das empresas. Verificou que índices como o endividamento e o percentual de despesas administrativas em relação às despesas totais eram positivamente associados à rentabilidade, enquanto a sinistralidade, a liquidez e a alavancagem demonstraram-se negativamente associadas à rentabilidade.

Ambrose \& Drennan (1994) desenvolveram um modelo logit para estimar a insolvência de uma OPS, focando as características observáveis dos planos, da região de atuação e do ambiente regulatório. Assumindo a igualdade de erros, o seu modelo previu corretamente a situação de solvência de aproximadamente $75 \%$ das operadoras da amostra. No entanto, a validação do modelo em outra amostra não foi testada.

Ahern e seus co-autores (AHERN, ROSENMAN e outros, 1996) analisaram as OPS da Flórida, e seu ambiente competitivo entre 1991-1993. Entre as variáveis estudadas estavam a natureza dos acionistas (hospital, médico, seguradoras, empresas), sua natureza jurídica (com e sem fins lucrativos), modalidade (autogestão, de grupo/rede, associações de prática independente, misto), tempo de plano (para refletir experiência), razão entre médicos generalistas e especialistas, concorrência (número de OPS na área de cobertura), taxa de ocupação hospitalar, características da população na área coberta (inclusive a densidade populacional e o número de habitantes acima de 65 anos) e a quantidade de médicos per capita na área de cobertura. Eles encontraram indícios de que a propriedade, a modalidade, e a natureza jurídica afetam a eficiência da OPS na prestação de serviços de saúde, principalmente pela alteração das diárias hospitalares.

Feldman, Wholey e Christianson (1996) analisaram dados de todas as OPS em operação nos Estados Unidos entre 1986 e 1993, e constataram que 80 OPS passaram por fusões e 149 haviam falido durante esse período. Desenvolveram um modelo logit, incluindo variáveis de mercado e a regulação antitruste, para prever se uma OPS iria se fundir e sobreviver, fundir e desaparecer ou falir em relação à probabilidade de qualquer outro evento. Seus resultados indicam que o tamanho da carteira de benefici- 
ários e a rentabilidade ajudam a explicar as fusões e ruínas de OPS, e que as empresas grandes e lucrativas têm mais probabilidade de sobreviver em uma fusão.

O presente estudo tem como foco a previsão de insolvência em um ano de uma OPS com base em indicadores financeiros. Vale notar que a literatura avaliada sobre previsão de insolvência de OPS oferece poucos estudos baseados em dados contábeis e medidas financeiras. Alguns analistas (AHERN, 1991) opinaram contra o uso de indicadores financeiros como o único instrumento de previsão de insolvência em virtude da falta de confiabilidade das informações. No entanto, qualquer variável está sujeita à falta de confiabilidade das informações, não somente a financeira. De fato, as variáveis financeiras podem, em certos casos, ser mais precisas que outros tipos de variáveis porque estão sujeitas à auditoria externa e ao controle de vários interessados, como: acionistas, reguladores e consumidores. Isso não quer dizer que não possa haver inconsistências em dados financeiros, ou que esses dados são necessariamente melhores, ou que não estejam sujeitos a fraudes. Como alguns casos de grande repercussão na mídia (Enron, Worldcom) corroboram, apesar do controle externo, nem sempre os dados são confiáveis. Outra vantagem importante dos indicadores econômicofinanceiros, além da disponibilidade dos dados e relação direta com a questão de estudo, é que sua interpretação é objetiva e bem conhecida, o que torna sua aplicação acessível a um público maior.

\section{A metodologia Cumulative Accuracy Profile (CAP)}

A CAP é uma metodologia de avaliação bastante utilizada atualmente para medir o desempenho de um sistema de classificação de risco de crédito. Para isso, os percentuais acumulados de contratos por classificação de risco são plotados contra os percentuais acumulados de inadimplentes em uma curva de Lorenz, com o objetivo de visualizar a acurácia da classificação. A curva foi desenvolvida por Max Lorenz e representa graficamente a proporcionalidade de uma distribuição.

Para construir a curva de Lorenz, as observações são ordenadas da maior para a menor probabilidade de insolvência (ou do menor para o maior escore Z", por exemplo). Se a classificação de insolvência é aleatória, a curva deverá se aproximar da diagonal, e o coeficiente de Gini

Figura 1 - A curva de Lorenz (CAP) e o coeficiente de Gini (AR)

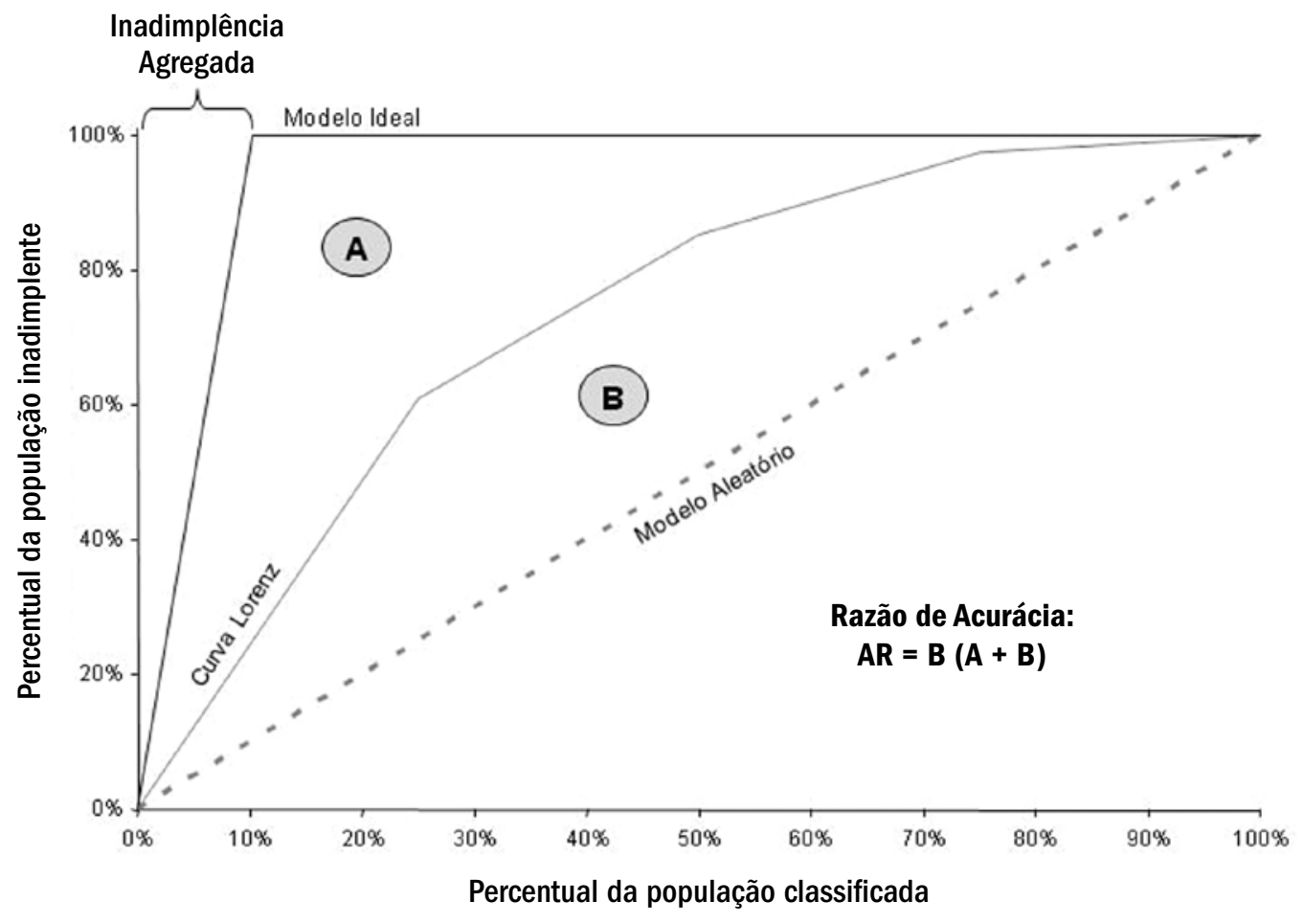

Fonte: Elaboração própria, a partir de Zazzarelli (2007). 
- que é a estatística de resumo da curva de Lorenz - deve ser igual a zero. Um modelo perfeito de classificação de risco tem o coeficiente de Gini igual a 1.

Com base na curva da Figura 1, o coeficiente de Gini - também chamado de accuracy ratio (AR), ou razão de acurácia - é calculado pela divisão da área $B$ pela área do triângulo $(\mathrm{A}+\mathrm{B})$. Em outras palavras, o coeficiente de Gini capta o quanto a acurácia da classificação de risco diverge de um cenário aleatório, ou ainda a capacidade do modelo em maximizar a distância entre clientes inadimplentes e adimplentes.

$\mathrm{Na}$ prática, as razões de acurácia típicas de sistemas de classificação de crédito encontram-se entre 50\% e 90\%, mas, além disso, pouco se pode dizer sobre a acurácia que um bom sistema deveria atingir (LOEFFLER e POSCH, 2007, p. 155). O sistema de classificação de crédito da Moody's, por exemplo, reporta uma razão de acurácia média de 74\% na América Latina, e 85\% globalmente (ZAZZARELLI, 2007).

Neste estudo, fazemos uma breve introdução à metodologia CAP. Outros autores (KEENAN e SOBEHART, 1999; SOBEHART, KEENAN E STEIN, 2000; SOBEHART e KEENAN, 2001; ENGELMANN, HAYDEN e TASCHE, 2003; ENGELMANN, HAYDEN, TASHCE e outros, 2003; SÁ FREIRE FILHO, BRANDI e outros, 2004; BASLE COMMITTEE ON BANKING SUPERVISION, 2005) abordam mais detalhadamente a metodologia CAP, comparando-a, inclusive, com outros modelos, como a curva ROC (receiver operating characteristic).

\section{METODOLOGIA}

Esta seção utiliza dados financeiros de OPS em 2004 e seu estado de solvência em 2005 (solvente $=0$, insolvente $=1$ ), a fim de desenvolver um modelo de regressão logística para estimar a probabilidade de insolvência de uma OPS em um ano. O modelo desenvolvido é validado numa amostra referente aos dados de OPS em 2005 e seu estado de solvência em 2006. Finalmente, o desempenho do modelo é comparado com o de um modelo de previsão de insolvência geral bastante difundido - o escore Z" de Altman.

\section{Amostra e ajustes}

As OPS brasileiras cobrem mais de 40 milhões de indivíduos. Esse mercado é regulado pela Agência Nacional de Saúde Suplementar (ANS). Os normativos vigentes estabelecem, entre outras coisas, a cobertura mínima que todas as OPS devem oferecer a seus clientes e um plano de contas contábil uniforme que deve ser adotado por todas as empresas. Além disso, a ANS dá publicidade aos demonstrativos financeiros e outras informações cadastrais apresentadas pelas OPS brasileiras (ANS, 2006). Os dados disponíveis sobre mais de 1.000 OPS, com cobertura razoavelmente uniforme (isto é, compreendendo um rol mínimo de procedimentos determinado pela ANS) e seguindo os mesmos padrões de contabilidade, são bastante adequados à análise aqui proposta. A partir desses dados, selecionamos apenas empresas que oferecem planos de assistência médica e hospitalar, deixando de lado aproximadamente 300 empresas dedicadas a planos exclusivamente odontológicos. Essa restrição foi imposta, principalmente, para permitir uma melhor comparabilidade dos dados em nossa amostra.

Antes da análise estatística, verificamos a consistência dos dados. Observações que impossibilitavam o cálculo de indicadores financeiros ou que apresentavam valores extremos foram descartadas. Por fim, por ser desnecessário prever a insolvência de empresas que já se apresentam como insolventes (com patrimônio líquido menor ou igual a zero), esses casos foram retirados de ambas as amostras (tanto de desenvolvimento quanto de validação do modelo). A amostra resultante - compreendendo 17 indicadores financeiros e 597 OPS - é descrita na Tabela 1. Vale notar que o tamanho da amostra e o número de observações por variável dependente excedem o mínimo recomendado de 100 e 10 casos, respectivamente (HARRELL, LEE e outros, 1984).

\section{ANÁLISE DOS DADOS}

\section{Variável dependente}

Neste estudo, considera-se a empresa insolvente quando o valor de seu patrimônio líquido, após um ano, é inferior ou igual zero. Assim, a amostra utilizada para a construção do modelo baseia-se nos dados financeiros referentes a 2004, e o estado de solvência ou insolvência (respectivamente, 0 e 1) em 2005, enquanto a amostra utilizada para a validação do modelo baseia-se nos dados financeiros de 2005, e seu estado de solvência ou insolvência em 2006.

\section{Variáveis independentes}

Existe um grande número de possíveis indicadores citados na literatura como úteis para prever dificuldades financeiras. Seguindo Altman \& Sabato (2007), iniciamos nossa análise com cinco categorias de indicadores contábeis que descrevem os principais aspectos da situação econômico-financeira de uma empresa: liquidez, renta- 
bilidade, alavancagem, cobertura e atividade. Cada uma das cinco categorias contém uma série de indicadores financeiros identificados na literatura como sendo mais bem-sucedidos na previsão de dificuldades financeiras (ver Quadro 1). Um aprofundamento sobre a interpretação dos indicadores listados acima está fora do escopo deste trabalho, mas os interessados encontrarão amplo material em textos especializados em administração financeira (ROSS, WESTERFIELD e outros, 2002; GITMAN, 2004).

A fim de evitar a multicolinearidade dos dados, que poderia afetar negativamente o modelo logístico binário, as variáveis que apresentaram correlação muito alta (acima de 0,80 ) foram retiradas da análise.

\section{RESULTADOS}

\section{Regressão logística}

Em seguida, aplicamos uma regressão logística binária. Para uma introdução ao modelo logístico, ver Hair Jr.,
Anderson, Tatham e Black (2005, p. 260-266), e um tratamento mais detalhado em Hosmer e Lemeshow (1989). A seleção escolhida foi a backward stepwise (em detrimento da forward stepwise, de modo a evitar efeitos supressores), deixando no modelo as variáveis com alfa abaixo de 0,05e retirando do modelo as variáveis com alfa superior a 0,1 . O modelo resultante (ver estatísticas na Tabela 2, e um resumo prático do modelo no Quadro 2) identificou três variáveis que, em conjunto, prevêem melhor a insolvência das OPS, com um ano de antecedência.

O sinal das três variáveis no modelo segue as expectativas, verificando-se uma relação positiva entre a situação de insolvência e os indicadores LnV01 - Ln(Passivo Circulante / Patrimônio Líquido) - e V13 (Receita Total/ Ativo Total), e uma relação negativa entre o indicador V09 (Resultado Líquido/Ativo Total) e a situação de insolvência, além disso o teste Wald para cada um dos preditores é estatisticamente significativo, o que quer dizer que os coeficientes são significativamente diferentes de zero. Além disso, o teste da razão das verossimilhanças

\section{Tabela 1 - Estatística descritiva}

\begin{tabular}{|c|c|c|c|c|c|}
\hline \multirow[t]{2}{*}{ CÓDIGO } & \multirow[t]{2}{*}{ VARIÁVEL } & \multicolumn{2}{|c|}{$\begin{array}{l}\text { SOLVENTES } \\
(\mathrm{N}=570)\end{array}$} & \multicolumn{2}{|c|}{$\begin{array}{c}\text { INSOLVENTES } \\
(\mathrm{N}=27)\end{array}$} \\
\hline & & MÉDIA & $\begin{array}{l}\text { DESVIO } \\
\text { PADRÃOO }\end{array}$ & MÉDIA & $\begin{array}{l}\text { DESVIO } \\
\text { PADRÃOO }\end{array}$ \\
\hline $\mathrm{V}_{01}$ & Passivo Circulante / Patrimônio Líquido & 1,9896 & 2,6919 & 11,2558 & 27,4297 \\
\hline $\mathrm{V}_{02}$ & Patrimônio Líquido / Exigivel Total & 1,0575 & 1,2160 & ,3555 & ,6069 \\
\hline $\mathrm{V}_{03}$ & Exigivel Total / Ativo Total & ,5895 & 2103 & ,7987 & , 1870 \\
\hline $\mathrm{V}_{04}$ & Patrimônio Líquido / Ativo Total & ,1116 & 1485 & 0955 & 1786 \\
\hline $\mathrm{V}_{05}$ & Disponível / Ativo Total & ,1250 & ,2527 &,- 0796 & ,2939 \\
\hline $\mathrm{V}_{06}$ & Capital de Giro / Ativo Total &, 0959 &, 2847 &, 0472 & ,0967 \\
\hline $\mathrm{V}_{07}$ & Disponível / Receita Total & ,4920 & ,3315 & ,4967 & ,4037 \\
\hline $\mathrm{V}_{08}$ & Resultado Antes de Impostos e Participações / Ativo Total &, 0639 &, 1213 &,- 0496 & ,2280 \\
\hline $\mathrm{V}_{09}$ & Resultado Líquido / Ativo Total &, 0481 & ,1039 &,- 0573 & ,2229 \\
\hline$V_{10}$ & Patrimônio Líquido / Ativo Total & ,4064 & ,2102 & ,1870 & ,1717 \\
\hline $\mathrm{V}_{11}$ & (Receitas Financeiras - Despesas Financeiras) / Receita Total & ,0002 & ,0389 &,- 0156 & ,0273 \\
\hline $\mathrm{V}_{12}$ & (Receitas Financeiras - Despesas Financeiras) / Ativo Total & 0087 & ,0455 &,- 0210 & ,0695 \\
\hline $\mathrm{V}_{13}$ & Receita Total / Ativo Total & 2,3114 & 1,5165 & 3,2038 & 3,1123 \\
\hline $\mathrm{V}_{14}$ & Passivo Circulante / Receita Total & ,4411 & 1,0785 & ,4706 &, 5336 \\
\hline$V_{15}$ & (Ativo Circulante + Investimentos de Longo Prazo) / Exigível Total & 1,0617 & ,7414 & 8440 & ,6618 \\
\hline $\mathrm{V}_{16}$ & (Disponibilidades - Empréstimos) / Ativo Total & ,2485 & ,2459 & 1513 & ,2712 \\
\hline$V_{17}$ & Capital de Giro / Receita Total & 0771 & ,7185 &,- 1281 & ,4126 \\
\hline
\end{tabular}


(Log-likelihood ratio) é estatisticamente significativo, ou seja, podemos afirmar que existe uma relação significativa entre o conjunto de variáveis selecionadas e o evento de insolvência. A estatística do teste Hosmer-Lemeshow de bondade de ajuste (HOSMER e LEMESHOW, 1989) não é significativa (ou seja, $\chi^{2}(8)=5,369, \mathrm{p}=0,717$ ), o que implica que as estimativas do modelo aderem aos dados de forma aceitável.

\section{DISCUSSÃO}

\section{Validação do modelo}

Com base nos dados financeiros de 2005, utilizamos uma amostra teste de 745 OPS, inclusive 20 insolventes, a fim de validar o desempenho do modelo desenvolvido especificamente para OPS e comparar seu desempenho ao de um modelo geral de previsão de insolvência (ver Tabela

\section{Quadro 1 - Variáveis consideradas}

\begin{tabular}{|c|c|c|}
\hline CATEGORIA DE INDICADOR & VARIÁVEL & FÓRMULA \\
\hline \multirow{5}{*}{ Alavancagem } & $\mathrm{V}_{01}$ & Passivo Circulante / Patrimônio Líquido \\
\hline & $\operatorname{LnV}_{01 *}$ & Ln(Passivo Circulante / Patrimônio Líquido) \\
\hline & $\mathrm{V}_{02}$ & Patrimônio Líquido / Exigivel Total \\
\hline & $\mathrm{V}_{03}$ & Exigivel Total / Ativo Total \\
\hline & $\mathrm{V}_{04}$ & Patrimônio Líquido / Ativo Total \\
\hline \multirow{4}{*}{ Liquidez } & $\mathrm{V}_{05}$ & Disponivel / Ativo Total \\
\hline & $\mathrm{V}_{06}$ & Capital de Giro / Ativo Total \\
\hline & $\mathrm{V}_{17}$ & Capital de Giro / Receita Total \\
\hline & $\mathrm{V}_{07}$ & Disponível / Receita Total \\
\hline \multirow{3}{*}{ Lucratividade } & $\mathrm{V}_{08}$ & Resultado Antes de Impostos e Participações / Ativo Total \\
\hline & $\mathrm{V}_{09^{*}}$ & Resultado Líquido / Ativo Total \\
\hline & $\mathrm{V}_{10}$ & Patrimônio Líquido / Ativo Total \\
\hline \multirow{3}{*}{ Atividade } & $\mathrm{V}_{13^{*}}$ & Receita Total / Ativo Total \\
\hline & $V_{14}$ & Passivo Circulante / Receita Total \\
\hline & $\mathrm{V}_{15}$ & (Ativo Circulante + Investimentos de Longo Prazo) / Exigível Total \\
\hline \multirow{3}{*}{$\begin{array}{l}\text { Endividamento } \\
\text { e Cobertura }\end{array}$} & $V_{11}$ & (Receitas Financeiras - Despesas Financeiras) / Receita Total \\
\hline & $\mathrm{V}_{12}$ & (Receitas Financeiras - Despesas Financeiras) / Ativo Total \\
\hline & $V_{16}$ & (Disponibilidades - Empréstimos) / Ativo Total \\
\hline
\end{tabular}

Tabela 2 - Estatísticas do modelo logístico para a previsão de insolvência de OPS

\begin{tabular}{|c|c|c|c|c|c|c|c|c|}
\hline VARIÁVEL & B & $\begin{array}{c}\text { ERRO } \\
\text { PADRÃO }\end{array}$ & $\begin{array}{c}\text { ESTATÍSTICA } \\
\text { WALD }\end{array}$ & $\begin{array}{c}\text { GRAUS DE } \\
\text { LIBERDADE }\end{array}$ & SIG. & EXP(B) & \multicolumn{2}{|c|}{ INT. CONF. 95\% P/ EXP(B) } \\
\hline LnV $_{01}$ & 1,206 &, 231 & 27,307 & 1 &, 000 & 3,340 & 2,125 & 5,250 \\
\hline$V_{09}$ & $-5,716$ & 1,539 & 13,787 & 1 &, 000 &, 003 &, 000 &, 067 \\
\hline$V_{13}$ &, 242 &, 109 & 4,937 & 1 &, 026 & 1,274 & 1,029 & 1,576 \\
\hline Constante & $-4,834$ &, 556 & 75,598 & 1 &, 000 &, 008 & - & - \\
\hline
\end{tabular}

Nota: -2 Log-likelihood $=159,764 ; R^{2}$ de Cox \& Snell $=0,096 ; R^{2}$ de Nagelkerke $=0,311$. 
3). A comparação da amostra de desenvolvimento incluiu 706 planos de saúde, inclusive 28 insolventes. Vale notar que, para fins de comparação de desempenho, a validação do modelo foi realizada nas amostras originais, incluindo aqueles dados excluídos no desenvolvimento do modelo (valores extremos), daí o maior número de casos quando comparado com o número de casos na Tabela 1.

A Tabela 3 resume os resultados, em termos de precisão, dos dois modelos, permitindo comparar o modelo logístico específico para OPS ao modelo geral de previsão de insolvência - o escore Z" desenvolvido por Altman (ALTMAN, BAIDYA e outros, 1979; ALTMAN e HOTCHKISS, 2006). Destaca-se que a razão de acurácia verificada na aplicação do modelo de escore Z" aos dados de OPS $(68,77 \%)$ é praticamente idêntica à verificada em um teste com dados de micro, pequenas e médias empresas (ALTMAN e SABATO, 2007).

A fim de comparar os dois modelos, destacamos dois índices na Tabela 3. O primeiro índice, apresentado na coluna 4, mede a precisão de cada modelo em classificar corretamente solvência e insolvência das empresas, e corresponde a um menos a média das taxas de erro tipo I e tipo II (assume pesos iguais para erros tipo I e II). O segundo índice, apresentado na coluna 5, é a chamada de razão de acurácia (AR) (ENGELMANN, HAYDEN e outros, 2003), e mede a capacidade do modelo em maximizar

\section{Quadro 2 - Um modelo logístico para a previsão de insolvência de OPS: resumo e aplicação}

Resumo do modelo logístico:

$\log \left(\frac{P I}{1-P I}\right)=-4,834+1,206 \times \log \left(\frac{P C}{P L}\right)-5,716 \times \frac{L L}{A T}+2,42 \times \frac{R T}{A T}$

Onde:

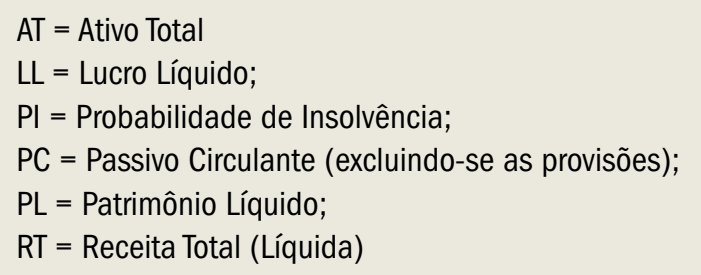

Valores sugeridos para corte:

Vermelho (alta probabilidade de insolvência): maior que 0,040

Amarelo (alguma probabilidade de insolvência): entre 0,025 e 0,040

Verde (insolvência improvável): $\quad$ menor que 0,025

\section{Aplicação:}

0 modelo pode ser implementado em planilha eletrônica, com a fórmula:

$=1 /\left(1+\operatorname{EXP}(1)^{\wedge}-\left[-4,834+1,206 * \mathrm{LN}(\mathrm{PC} / \mathrm{PL})-5,716^{*} \mathrm{LL} / \mathrm{AT}+0,242 * \mathrm{RT} / \mathrm{AT}\right]\right.$.

Tabela 3 - Comparação da acurácia dos modelos analisados

\begin{tabular}{|c|c|c|c|c|c|}
\hline $\begin{array}{l}\text { AMOSTRA } \\
\text { (ANO) }\end{array}$ & MODELO & $\begin{array}{l}\text { TAXA DE } \\
\text { ERRO } \\
\text { TIPO I* }\end{array}$ & $\begin{array}{l}\text { TAXA DE } \\
\text { ERRO } \\
\text { TIPO II * }\end{array}$ & $\begin{array}{c}1 \text { - MÉDIA DAS } \\
\text { TAXAS DE ERRO } \\
\text { TIPOS I E II * }\end{array}$ & $\begin{array}{l}\text { RAZÃO DE } \\
\text { ACURÁCIA } \\
* *\end{array}$ \\
\hline Desenvolvimento & Logit específico para OPS & $17,86 \%$ & $21,39 \%$ & $80,38 \%$ & $71,60 \%$ \\
\hline (2004) & Modelo geral: escore Z" de Altman & $46,43 \%$ & $22,57 \%$ & $65,50 \%$ & $36,22 \%$ \\
\hline Teste & Logit específico para OPS & $23,58 \%$ & $25,00 \%$ & $75,71 \%$ & $69,65 \%$ \\
\hline (2005) & Modelo geral: escore Z" de Altman & $23,58 \%$ & $25,00 \%$ & $75,71 \%$ & $68,77 \%$ \\
\hline
\end{tabular}

* Ponto de corte em 25\% do tamanho da amostra.

** Independente do ponto de corte. 
a distância entre solventes e insolventes. As curvas CAP para os dois modelos são apresentadas nas figuras 2 e 3 .

Os coeficientes de erro apresentados na Tabela 5 são calculados pela determinação de um ponto de corte arbitrário de $25 \%$ das amostras e são muito próximos daqueles obtidos a partir da amostra de desenvolvimento (ver

Figura 2 - Comparação da acurácia do modelo logístico específico para OPS e do modelo de escore Z" de Altman na amostra de desenvolvimento pela metodologia CAP

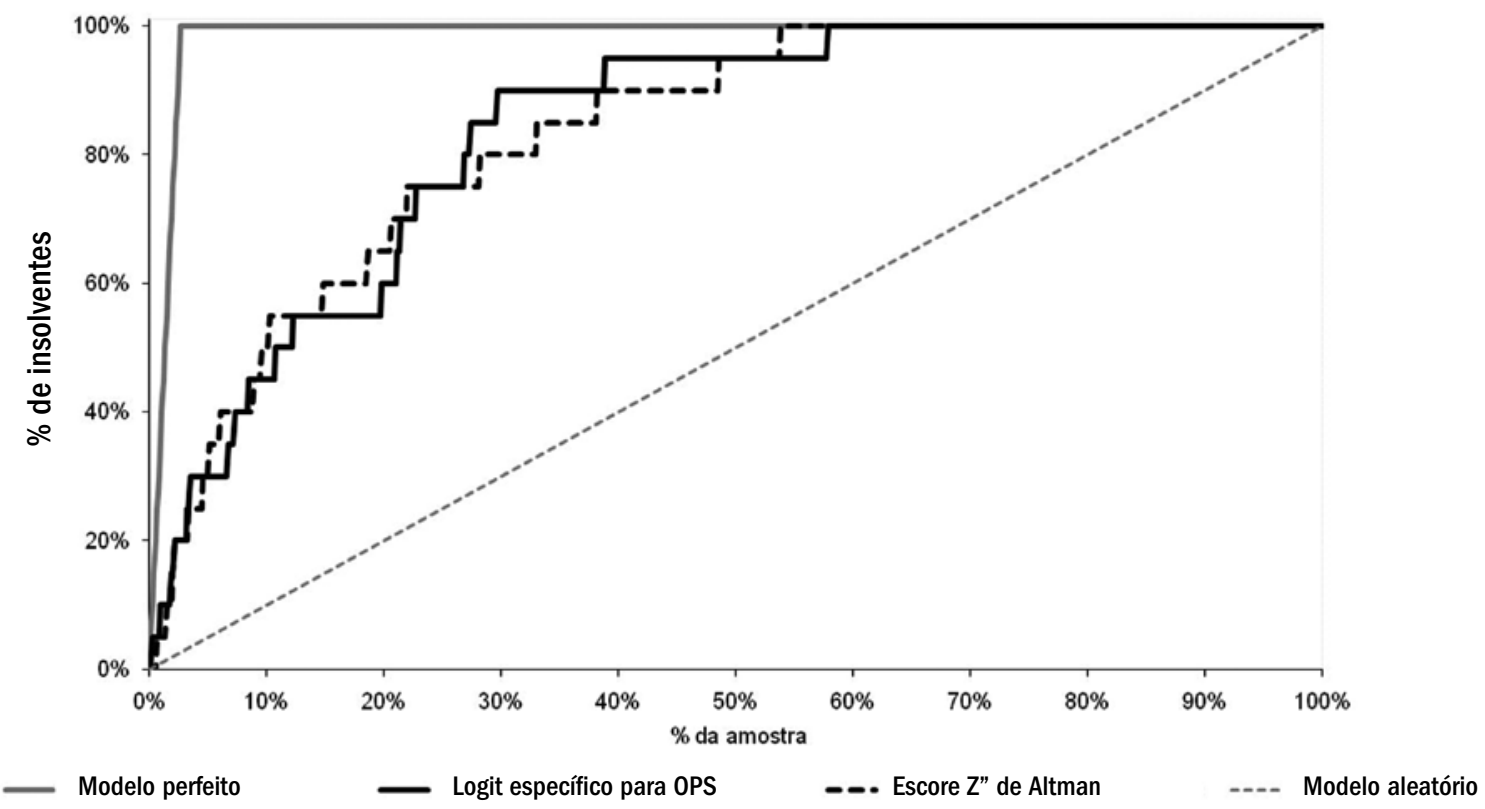

Figura 3 - Comparação da acurácia do modelo logístico específico para OPS e do modelo de escore Z" de Altman na amostra de teste pela metodologia CAP

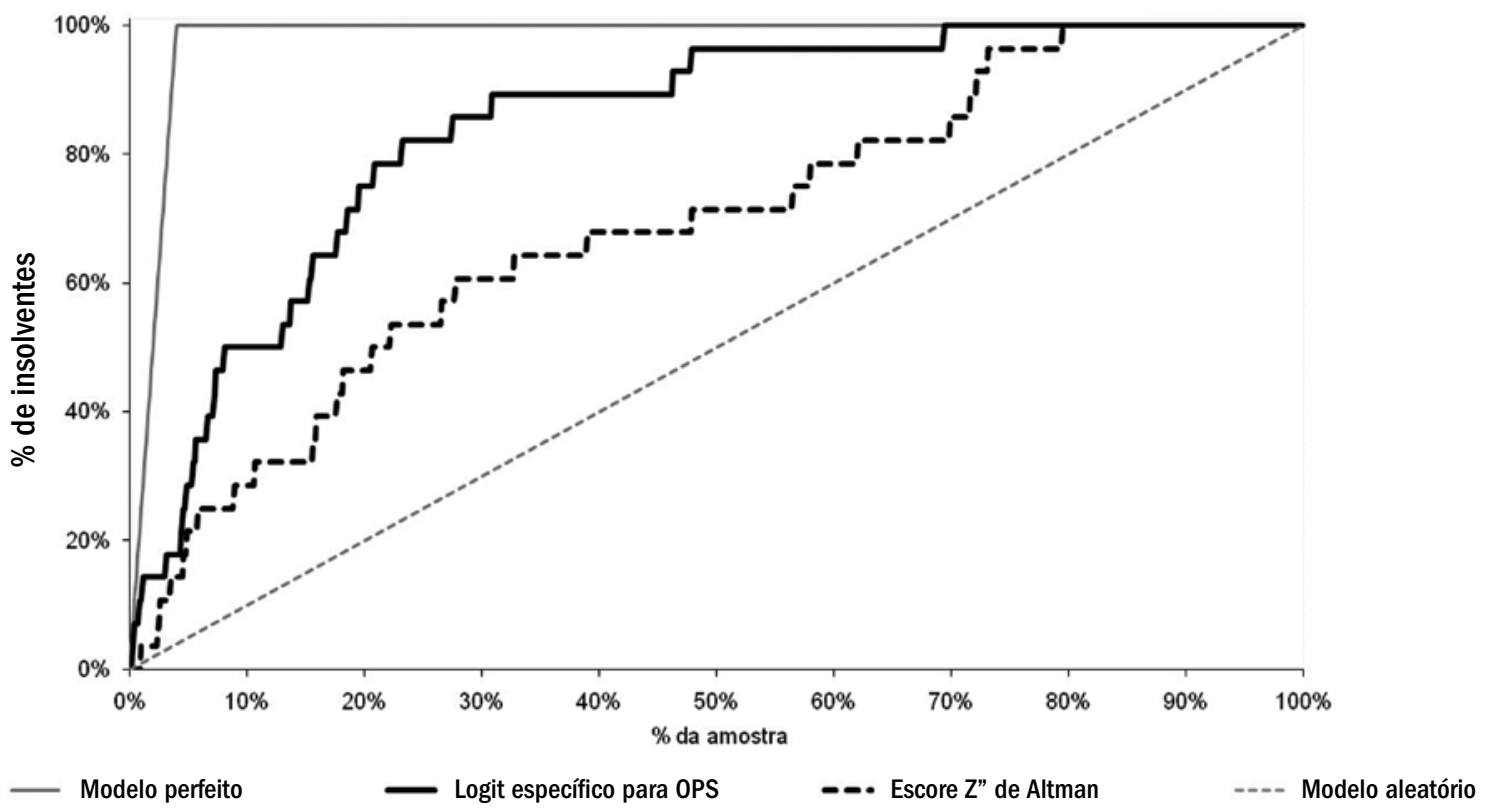


Tabela 5). O indicador de precisão (AR) do nosso modelo específico para OPS foi de $71,60 \%$ para a amostra de treino e $69,65 \%$ para a amostra de teste, em comparação com, respectivamente, 36,22 e 68,77 do modelo geral de escore Z". É importante destacar, porém, que o indicador de acurácia não depende do ponto de corte escolhido, pois avalia o modelo em relação a todos os resultados apurados na amostra analisada.

Sabemos que todo modelo é uma aproximação imperfeita da realidade. Como a crise econômica mundial ocorrida no final de 2008 deixou claro, modelos de previsão de insolvência e de classificação de risco não escapam a essa regra. Testando o modelo apresentado em 2006/2007 (estimando com base nos demonstrativos contábeis de 2006, a probabilidade de insolvência em 2007) numa amostra de 904 operadoras (26 das quais se tornaram insolventes em 2007) observamos uma razão de acurácia de 62,27\% em 2006/2007. No ano seguinte (2007/2008), aplicando o modelo numa amostra de 775 operadoras ( 24 das quais se tornaram insolventes em 2008), observamos uma razão de acurácia de 65,09\%. Tais resultados são bastante satisfatórios considerando-se o cenário econômico e o impacto de medidas regulatórias (aumento das provisões, registro definitivo) sobre as médias amostrais.

Podemos afirmar que o modelo específico para OPS é estatisticamente robusto e válido, uma vez que sua precisão permanece elevada em ambas as amostras. Verificamos, porém, que o modelo geral de previsão de insolvência mostrou uma variação significativa na precisão (a grande diferença de precisão para as amostras de desenvolvimento e teste), sugerindo que sua robustez e confiabilidade pode ser limitada. Reconhecemos que o ponto de corte selecionado pode não ser ótimo, principalmente se forem considerados diferentes pesos para erros do tipo I e tipo II (ALTMAN, HALDEMAN e outros, 1977). No entanto, o objetivo desta comparação não é determinar o ponto de corte ótimo, mas apenas utilizar o mesmo ponto de corte a fim de comparar a precisão de modelos diferentes.

A escolha de um ponto de corte envolve um trade-off entre taxas de erros do tipo I e tipo II aceitáveis. Esta seleção pode ser vista como estratégica e está relacionada com a aversão a risco do analista. As figuras 4 e 5 apresentam um close-up do desempenho dos modelos em diferentes pontos de corte, e pode servir como uma referência para a seleção dos pontos de corte para os dois modelos.

Para o modelo logit específico para OPS, como mostra a Figura 4, um ponto de corte de 0,04 apresenta resultado bastante próximo da classificação apresentada na Tabela 5 (valores superiores a 0,04 têm uma elevada probabilidade de insolvência).

Enquanto isso, como vemos na Figura 5, para o escore Z" de Altman, um ponto de corte com poder discriminan-

Figura 4 - Desempenho em diferentes pontos de corte: Logit específico para OPS

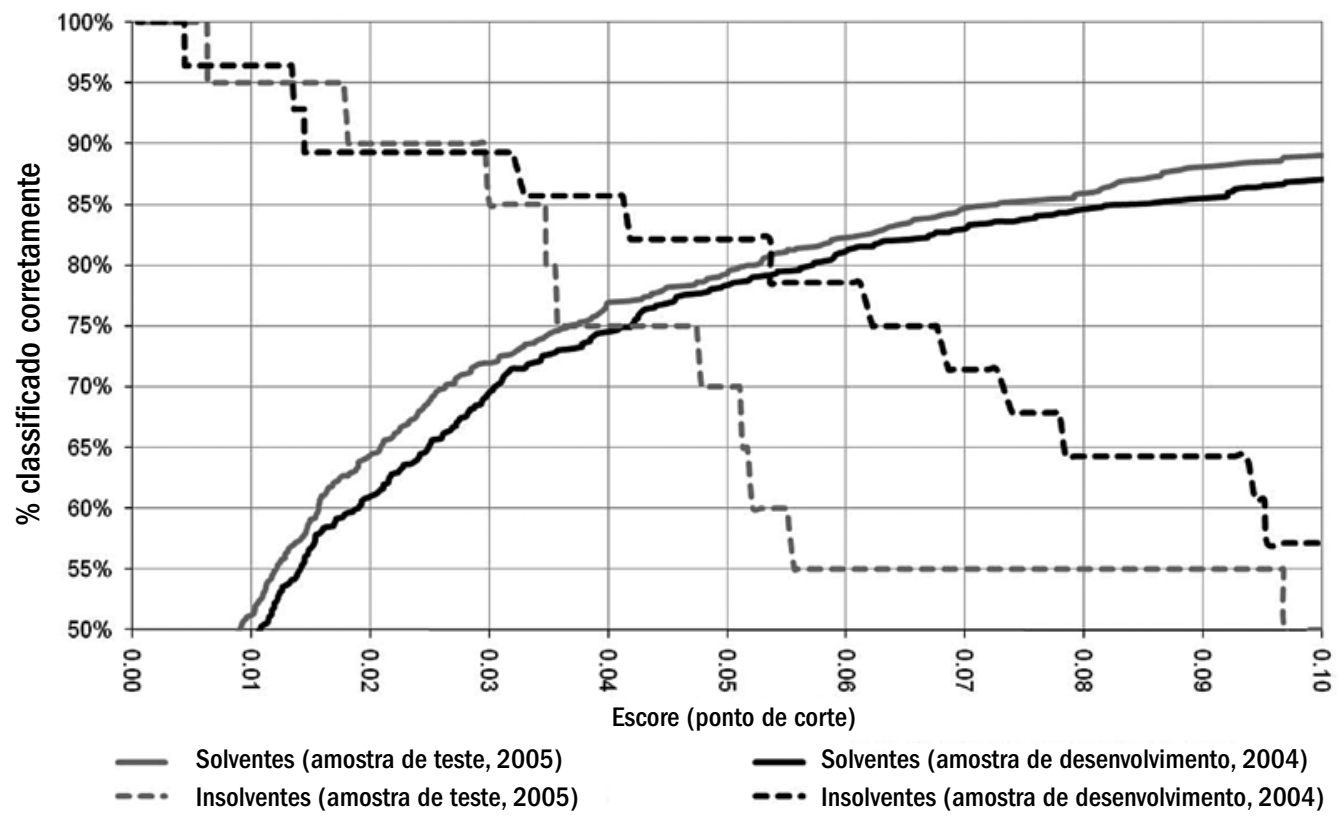


te próximo ao do modelo específico para OPS requer a adoção de um ponto de corte próximo a 4,00 (sendo os valores menores que 4,00 classificados como insolventes), um escore consideravelmente superior ao ponto de corte original do modelo (onde valores abaixo de 1,1 indicam insolvência).

\section{CONCLUSÃO}

Nesse artigo, desenvolvemos e testamos um modelo de previsão de insolvência para operadoras de planos de saúde. Ampliamos a literatura existente por considerar um conjunto completo de indicadores financeiros e obter um modelo de regressão logística para a estimativa da probabilidade de insolvência, em um ano, de operadoras de planos de saúde. Avaliamos o desempenho do modelo específico desenvolvido sobre uma amostra de validação comparando a sua precisão com a de um modelo geral de previsão de insolvência bastante conhecido, o escore Z" de Altman. Os resultados confirmam nossas expectativas. O desempenho, em termos de precisão, de um modelo específico para OPS é superior ao desempenho do modelo geral. Nossos resultados indicam, entretanto, que modelos gerais como o escore Z" de Altman podem ser eficazes se utilizarmos um valor diferente como ponto de corte, embora sua confiabilidade (variação de desempenho para diferentes amostras) seja inferior à do modelo específico. Nossos resultados sugerem que a previsão de insolvência de operadoras de planos de saúde é mais precisa e confiável quando se utiliza um modelo específico. Vale destacar que modelos de previsão de insolvência com base em informações financeiras como o aqui apresentado têm sido aplicados a mais de 50 anos. Porém, sua utilidade torna-se ainda maior quando tomados em conjunto com outras formas de análise mais abrangentes (qualitativas, operacionais etc.). Finalmente, esperamos que estudos futuros possam desenvolver um modelo ainda mais abrangente, que incorpore medidas financeiras assim como características estruturais das operadoras e sua situação concorrencial.

\section{NOTA DE AGRADECIMENTO}

Esse artigo se beneficiou dos comentários de Dr. Alan B. Eisner e Dr. Raymond Lopez (Pace University), e de revisores anônimos da Academy of Management Conference 2009 e RAE-revista de administração de empresas. Eventuais erros e omissões são de responsabilidade dos autores.

Figura 5 - Desempenho em diferentes pontos de corte: escore Z" de Altman

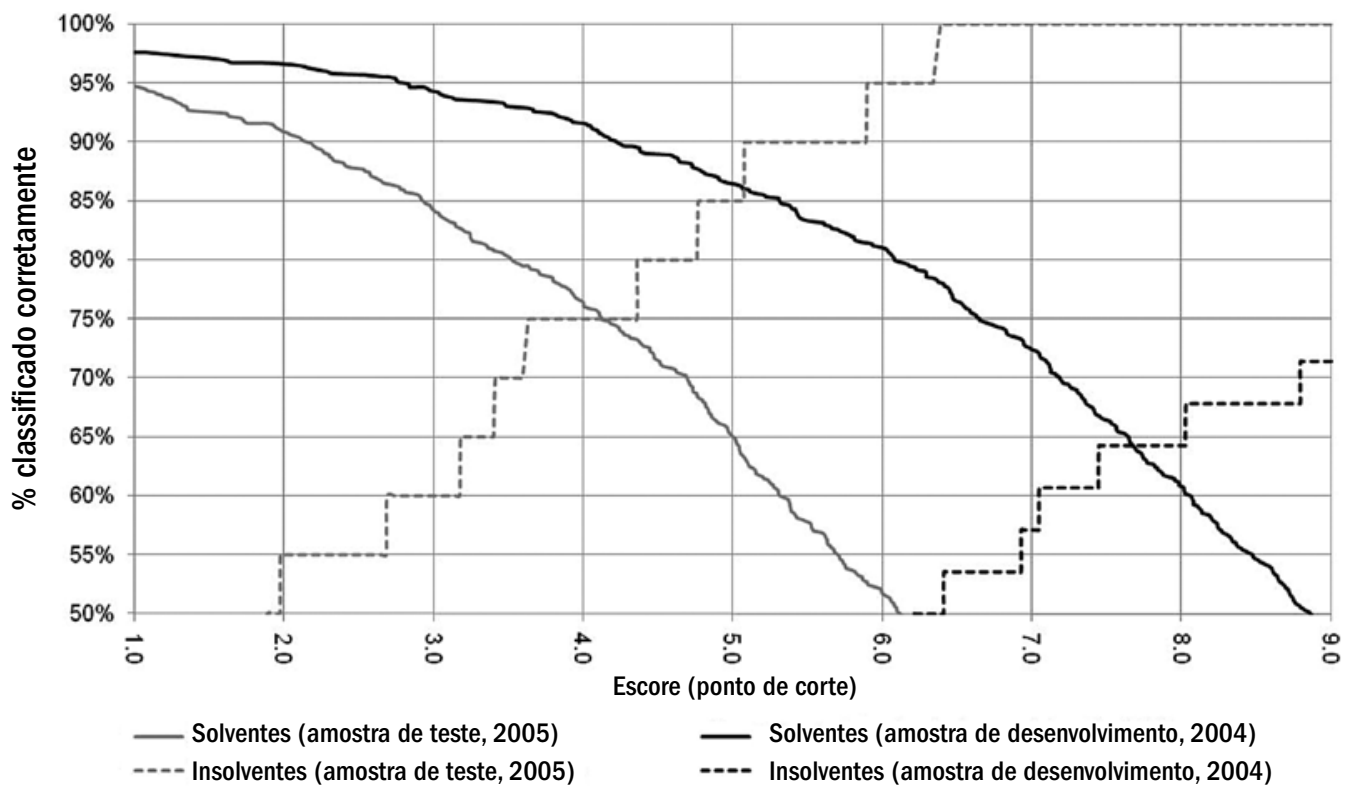




\section{REFERÊNCIAS}

AHERN, M. The evolution and impact of state HMO regulation: a case study. Journal of Insurance Regulation, v. 10, n. 1, p. 110-127, 1991.

AHERN, M; ROSENMAN, R. e outros. Predictors of HMO efficiency. Applied Economics, v. 28, n. 11, p. 1381-1390, 1996.

ALTMAN, E; HARTZELL, J; PECK, M. Emerging Markets Corporate Bonds: A Scoring System. New York: Salomon Brothers, Inc, 1995.

ALTMAN, E; HARTZELL, J; PECK, M. Emerging markets corporate bonds: a scoring system. In: LEVICH, R; MEI, J. P. (Ed). Future of Emerging Market Flows. Holland: Kluwer, 1997.

ALTMAN, E. I. Financial ratios, discriminant analysis and the prediction of corporate bankruptcy. Journal of Finance, v. 23, n. 4, p. 589, 1968/09 1968.

ALTMAN, E. I; BAIDYA, T. K. N; DIAS, L. M. R. Assessing Potential Financial Problems for Firms in Brazil. Journal of International Business Studies, v. 10, n. 2, p. 9, 1979

ALTMAN, E. I; HALDEMAN, R. G; NARAYANAN, P. Zeta analysis: a new model to identify bankruptcy risk in corporation. Journal of Banking $\mathcal{E}$ Finance, v. 1, n. 1, p. 29-54, 1977.

ALTMAN, E. I; HOTCHKISS, E. Corporate Financial Distress and Bankruptcy: Predict and Avoid Bankruptcy, Analyze and Invest in Distressed Debt. 3. ed. Hoboken, NJ: John Wiley \& Sons, 2006.

ALTMAN, E. I; SABATO, G. Modeling credit risk for SMEs: evidence from the US market. Abacus, v. 43, n. 3, p. 332-357, 2007.

AMBROSE, J. M; DRENNAN, R. B. Plan, market and regulatory considerations in $\mathrm{HMO}$ insolvency prediction. Journal of Insurance Regulation, $\mathrm{v}$. 12, n. 3, 1994

ANUÁRIO ANS. Aspectos econômico-financeiros das operadoras de planos de saúde: 2005. Rio de Janeiro, Brasil: Agência Nacional de Saúde Suplementar, 2006. $624 \mathrm{p}$

BASLE COMMITTEE ON BANKING SUPERVISION. Studies on the Validation of Internal Rating Systems. Basle, SW: Bank for International Settlements, 2005

BEAVER, W. H. Financial ratios as predictors of failure. Journal of Accounting Research, v. 4, p. 71-111, 1966

CHRISTIANSON, J. B; WHOLEY, D. R; SANCHEZ, S. M. State responses to HMO failures. Health Affairs, v. 10, n. 4, p. 78, 1991.

ENGELMANN, B; HAYDEN, E; TASCHE, D. Measuring the Discriminative Power of Rating Systems. Discussion paper. Series 2: Banking and Financial Supervision. Frankfurt, Germany: Deutsche Bundesbank, 2003.

ENGELMANN, B; HAYDEN, E; TASCHE, D. Testing rating accuracy. Risk, v. 16, n. 1, p. 82-86, 2003.

FELDMAN, R; WHOLEY, D; CHRISTIANSON, J. Economic and organizational determinants of HMO mergers and failures. Inquiry, v. 33, n. 2 , p. 118-32, 1996
FITZPATRICK, P. J. A comparison of the ratios of successful industrial enterprises with those of failed companies. Certified Public Accountant, 1932

GITMAN, L. J. Princípios de administração financeira. 10a. ed. São Paulo: Addison Wesley, 2004. 745 p.

HAIR JR, J. F; ANDERSON, R. E. e outros. Análise multivariada de dados 5a. ed. Porto Alegre: Bookman, 2005.

HARRELL, F. E; LEE, K. L. e outros. Regression modelling strategies for improved prognostic prediction. Statistics in Medicine, v. 3, n. 2, p. 143 52,1984

HILLMAN, A. L; PAULY, M. V; KERSTEIN, J. J. How do financial incentives affect physicians' clinical decisions and the financial performance of health maintenance organizations? New England Journal of Medicine, v 321, n. 2, p. 86-92, 1989

HOSMER, D. W; LEMESHOW, S. Applied Logistic Regression. New York: John Wiley \& Sons, 1989

KEENAN, S; SOBEHART, J. Performance measures for credit risk models. Moody's Risk Management Services, Research Report, 1999.

LOEFFLER, G; POSCH, P. N. Credit Risk Modeling using Excel and VBA. Hoboken, NJ: John Wiley \& Sons, 2007.

OHLSON, J. A. Financial ratios and the probabilistic prediction of bankruptcy. Journal of Accounting Research, v. 18, n. 1, p. 109-131, 1980

ROSS, S. A; WESTERFIELD, R. W; JAFFE, J. F. Administração financeira. 2a. ed. São Paulo: Atlas, 2002. 776 p.

SÁ FREIRE FILHO, A. A; BRANDI, V. R. e outros. Aferindo o poder discriminatório dos modelos de classificação de risco de crédito. $10^{a}$ Semana de Contabilidade. Brasília, DF: Banco Central do Brasil, 2004.

SOBEHART, J; KEENAN, S. Measuring default accurately. Credit Risk Special Report. Risk, v. 14, p. 31-33, 2001.

SOBEHART, J; KEENAN, S; STEIN, R. Benchmarking quantitative default risk models: a validation methodology. Moody's Investors Service, Mar. 2000

STONE, D. L; HEFFERNAN, S. 1988 survey on regulation of HMOs and HMO solvency: findings and recommendations. GHAA Journal, v. 10, n. 1, p. 28-39, 1989.

WHOLEY, D; CHRISTIANSON, J; SANCHEZ, S. The effect of state regulation on development of HMO markets. Advances in the Study of Entrepreneurship, Innovation, and Economic Growth: A Research Annual, p. 181-206, 1990.

ZAZZARELLI, A. Latin American corporate default and recovery rates, 1990-H1 2007. Moody's Investor Service, Nov. 2007. 